\title{
Hsa_circ_0068307 mediates bladder cancer stem cell-like properties via miR-147/c-Myc axis regulation
}

\author{
Qi Chen ${ }^{1,2 \dagger}$, Qiuping Yin ${ }^{1,2+}$, Yemeng Mao ${ }^{3 \dagger}$, Zheyu Zhang ${ }^{1,2}$, Siqi Wu ${ }^{1,2}$, Zhang Cheng ${ }^{1,2}$, Xinan Chen ${ }^{1,2}$, \\ Hanren $\mathrm{Xu}^{4}$, Shengming Jin ${ }^{5}$, Haowen Jiang ${ }^{1,2,6^{*}}$ and Chen Yang ${ }^{1,2,6^{*}}$ (1)
}

\begin{abstract}
Background: Circular RNAs (circRNAs) play an essential role in the regulation of gene expression. However, the underlying mechanisms remain unknown. This study aimed to evaluate the role of hsa_circ_0068307 in bladder cancer (BCa).

Methods: Rt-qPCR was used to detect hsa_circ_0068307 expression in BCa cell lines. The CCK8, colony formation, and Transwell assays were used to evaluate the effect of hsa_circ_0068307 on BCa cell migration and proliferation. Bioinformatics and luciferase reporter experiments were used to study the regulatory mechanism. Nude mouse xenografts were generated to examine the effect of hsa_circ_0068307 on tumor growth.

Results: The results showed that hsa_circ_0068307 was upregulated in BCa cell lines. Downregulation of hsa_ circ_0068307 suppressed cell migration and proliferation in T24 and UMUC3 cells. Hsa_circ_0068307 silencing suppressed cancer stem cell differentiation by upregulating miR-147 expression. Upregulation of miR-147 suppressed c-Myc expression, which is involved in cancer stem cell differentiation. Luciferase reporter assays confirmed that hsa_circ_0068307 upregulated c-Myc expression by targeting miR-147. In vivo studies showed that hsa_circ_0068307 knockdown suppressed T24 tumor growth.
\end{abstract}

Conclusions: These data indicate that downregulation of hsa_circ_0068307 reversed the stem cell-like properties of human bladder cancer through the regulation of the miR-147/c-Myc axis.

Keywords: Hsa_circ_0068307, Bladder cancer, miR-147, c-Myc, Cancer stem cell

\section{Background}

Bladder cancer $(\mathrm{BCa})$ is a common malignancy worldwide, with 430,000 new cases diagnosed in 2012 [1]. In China, $\mathrm{BCa}$ was the sixth most common cancer among men in 2015 [2]. Approximately $75 \%$ of newly diagnosed BCas are non-muscle invasive, and $70 \%$ of them experience relapse, with $25 \%$ progressing to muscle-invasive

\footnotetext{
*Correspondence: Zhyishya@163.com; YangC_Huashan@163.com

${ }^{\dagger} \mathrm{Q}$ i Chen, Qiuping Yin and Yemeng Mao are co-first authors

1 Department of Urology, Huashan Hospital, Fudan University, Shanghai, China

Full list of author information is available at the end of the article
}

$\mathrm{BCa}$ [1]. Because of the high incidence, progression, and recurrence rates, the 5-year survival rate of $\mathrm{BCa}$ patients is low [3]. Therefore, elucidating the mechanisms that contribute to metastasis, progression, and development of $\mathrm{BCa}$ is indispensable.

Circular RNAs (circRNAs) are a newly discovered of endogenous noncoding RNAs (ncRNAs) constructed from introns or exons via particular selective shearing. Derived from linear RNA, circRNAs consist of covalently closed loop structures that have unique, highly stable, and diverse forms [4-6]. circRNAs have critical functions in many pathological and biological processes, including

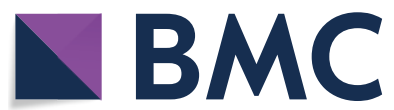

(c) The Author(s) 2020. This article is licensed under a Creative Commons Attribution 4.0 International License, which permits use, sharing, adaptation, distribution and reproduction in any medium or format, as long as you give appropriate credit to the original author(s) and the source, provide a link to the Creative Commons licence, and indicate if changes were made. The images or other third party material in this article are included in the article's Creative Commons licence, unless indicated otherwise in a credit line to the material. If material is not included in the article's Creative Commons licence and your intended use is not permitted by statutory regulation or exceeds the permitted use, you will need to obtain permission directly from the copyright holder. To view a copy of this licence, visit http://creativeco mmons.org/licenses/by/4.0/. The Creative Commons Public Domain Dedication waiver (http://creativecommons.org/publicdomain/ zero/1.0/) applies to the data made available in this article, unless otherwise stated in a credit line to the data. 
cell migration, invasion, and proliferation, cell cycle progression, metastasis, and carcinogenesis [7-10]. Previous studies showed that circCASC15 may function as a miR1224-5p sponge to activate CREB1 expression and promote cell proliferation in $\mathrm{BCa}$ [11]. CircHIPK3 sponges miR-558 to suppress $\mathrm{BCa}$ cell heparanase expression [12]. However, the role of hsa_circ_0068307 in BCa is unknown.

Cancer stem cells (CSCs) are important for tumor initiation and development [13]. CSCs express specific markers such as Oct4, Sox2, and NANOG [14]. Targeting CSCs is a promising and novel strategy for the treatment of tumors that are resistant to known therapies, which lead to treatment failure or recurrence [15]. Circ008913 participates in CSC-like property acquisition induced by arsenite through keratinocytes, thereby affecting carcinogenesis via miR-889 DAB2IP/ZEB1 regulation [16]. However, the role of hsa_circ_0068307 in the regulation of $\mathrm{BCa}$ development by CSCs remains unknown. The purpose of this study was to elucidate the hsa_circ_0068307 regulatory mechanism using in vitro and in vivo experiments.

\section{Materials and methods}

\section{Animal ethics statement}

Four-week-old nude BALB/c mice $(\mathrm{n}=12)$ weighing 15-20 g (SLARC, Shanghai, China) were used. The Ethics Committee of Huashan Hospital, Fudan University approved all animal experiments.

\section{Tissue samples}

In total, 30 fresh Bca and paired adjacent noncancerous $\mathrm{BCa}$ tissues were collected after obtaining informed consent from patients at Huashan Hospital, Fudan University. Samples were snap-frozen in liquid nitrogen and stored at $-80^{\circ} \mathrm{C}$ before RNA extraction. Huashan Hospital Ethics Committee in Fudan University approved this study.

\section{Cell culture and transfection}

BC and SV-HUC cell lines (EJ, T24, RT-4 and UM-UC3) were obtained from the Type Culture Collection of the Chinese Academy of Sciences, Shanghai and cultured at $37{ }^{\circ} \mathrm{C}$ in $5 \% \mathrm{CO}_{2}$ in DMEM medium (Gibco, Gaithersburg, MD, USA) containing 10\% fetal bovine serum (FBS; Gibco). T24 cells were transfected with miR-147 mimics, small interfering RNAs (siRNAs), miR-147 inhibitors, c-Myc overexpression vector, and negative controls using Lipofectamine 2000 (Invitrogen, Carlsbad, CA, USA), according to the standard procedure. Full length fragment of c-Myc was constructed into a GV358 vector to induce c-Myc overexpression (Genechem, Shanghai, China). To confirm the hsa_circ_0068307 effect in in vivo experiments, we constructed lentiviral stabilized hsa_circ_0068307 silenced T24 cells through PAX2/ PMD2G packing vectors, while shRNA targeting hsa circ_0068307 was cloned into PLKD-CMV-EGFP vectors (GeneWiz, Guangzhou, China).

si-NC sense: UUCUCCGAACGUGUCACGUTT.
si-NC antisense: ACGUGACACGUUCGGAGA
ATT.
si-circ sense: CUCAAGCAGUACACAGAGATT.
si-circ antisens: UCUCUGUGUACUGCUUGT
GTT.

\section{Migration assay}

Transwell chamber migration assays were performed. In brief, cells were resuspended with culture medium including 1\% FBS (Biological Industries, Cromwell, CT, USA), and $200 \mu \mathrm{L}$ cell suspension $\left(1 \times 10^{5}\right.$ cells $)$ was added into the Transwell chamber (BD Biosciences, Franklin Lakes, NJ, USA). The lower chamber was filled with $500 \mu \mathrm{L}$ medium containing FBS at $30 \%$. After incubation for 1 day at $37{ }^{\circ} \mathrm{C}$ with $5 \% \mathrm{CO}_{2}$, cells were rinsed with PBS and fixed in $0.5 \%$ methanol for $30 \mathrm{~min}$, then stained with crystal violet $0.1 \%$ for $20 \mathrm{~min}$, and rinsed with PBS. Non-invading cells were removed using a cotton swab, and migrating cells were observed by microscopy and counted. All experiments were performed in triplicate.

\section{Bioinformatics analysis}

We predicted circRNA/miRNA target genes via the online tool, Circular RNA Interactome. We predicted interacting relationships between miR-147 and c-Myc through the web-based package, TargetScanHuman.

\section{Cloning formation assay and cell proliferation assay}

The Cell Counting Kit-8 (CCK-8) assay was used to explore cell proliferation. Transfected cells were seeded into triplicate wells of 96-well plates at a density of 2000 cells/well. We measured cell viability via the CCK- 8 system (Gibco) at $0,24,48,72$, and $96 \mathrm{~h}$ after seeding, following the standard procedure.

Transfected cells were seeded into six-well plates at a density of 2000 cells per well in DMEM containing FBS at $10 \%$ for 10 days for the colony formation assay. Colonies were imaged and counted after fixing and staining.

\section{Western blots}

Protein was extracted from cells and tumor tissues using RIPA lysis buffer containing protease inhibitors (SigmaAldrich, St. Louis, MO, USA) and the BCA Protein Assay kit (Vigorous Biotechnology Beijing, Beijing, China) was 
used to measure protein concentration. Twenty micrograms of protein per sample were separated using SDSPAGE and transferred onto nitrocellulose membranes (Millipore, Madison, WI, USA), which were blocked with $5 \%$ nonfat dry milk for $2 \mathrm{~h}$ before incubation with primary antibodies at $4{ }^{\circ} \mathrm{C}$ overnight. Glyceraldehyde 3-phosphate dehydrogenase was used as the internal control. Membranes were incubated with horseradish peroxidase-conjugated secondary antibodies for $1 \mathrm{~h}$ at room temperature.

\section{RNA extraction and quantitative reverse}

\section{transcription-polymerase chain reaction (RT-qPCR)}

RNA extraction was performed using the TRIzol reagent (Invitrogen), as described in Li et al. [17]. cDNA was obtained using the pTRUEscript 1st Strand cDNA Synthesis Kit (Aidlab, Beijing, China). qRt-PCR was performed using the $2 \times$ SYBR Green qPCR Mix (Aidlab) on an ABI $7900 H T$ sequence detection instrument (Thermo Fisher Scientific, Waltham, MA, USA). Fold-changes in expression were calculated using the $2^{-\Delta \Delta \mathrm{CT}}$ method.

\section{Luciferase reporter assay}

Wild type (WT) hsa_circ_0068307 cDNA fragments, including the predicted miR-147 binding sites were amplified and mutated fragments (Mutant) were generated by overlap extension PCR. WT and mutant fragments were cloned into the psiCHECK-2 (Promega, Madison, WI, USA). HEK293T cells were co-transfected with WT or mutant vector and miR-147 or control mimics using Lipofectamine 2000 (Thermo Fisher Scientific) for luciferase reporter assays. After 2 days of transfection, luciferase activity was detected using a dual-luciferase reporter assay kit (Promega) and normalized to Renilla luciferase activity. All experiments were performed in triplicate.

\section{Metastasis assays and tumor xenograft formation}

In total, we injected $2 \times 10^{7}$ viable cells from wild-type or sh-circRNA T24 cells into the right flanks of 12 nude mice [18]. We measured tumor sizes every 5 days using a Vernier caliper. Tumor volume was calculated using the following formula: volume $=0.5 *$ width $^{2}$ * Length. Mice were euthanized for qRT-PCR analyses 1 month after implantation.

\section{Tumor sphere formation assay}

Cells were harvested and re-suspended as single cells in a non-serum medium (as previously mention in Tumor specimens and cell culture). After accurate cell counting, 200 cells/well in $200 \mu \mathrm{L}$ of non-serum medium were added to a 96 well plate, and each group was in 10 wells. The medium was change every 2 days. Image of five randomly selected regions of each group were taken with a microplate reader (Leica, Wetzlar, Germany). The sphere percentage was calculated as the number of sphere/200.

\section{Statistical analysis}

Data were expressed as the mean \pm standard deviation (SD). GraphPad Prism (version 5.0; GraphPad, La Jolla, CA, USA) was used to compare differences among groups. A $P$ value $\leq 0.05$ inferred statistical significance.

\section{Results}

Hsa_circ_0068307 is highly expressed in BCa and exerts oncogenic effects in UMUC3 and T24 BCa cell lines

Rt-qPCR detection showed that hsa_circ_0068307 expression was higher in $\mathrm{BCa}$ tissues than in adjacent normal tissues in our cohort (Fig. 1a). The results also showed that hsa_circ_0068307 expression was higher in the BCa cell lines EJ, RT-4, T24, and UMUC-3 than in SV-HUC-1 cells (Fig. 1b). Because, T24 and UMUC-3 cell have more higher hsa_circ_0068307 expression, so we selected T24 and UMUC-3 cells for further study. Cells were treated with siRNA against hsa_circ_0068307 (sicircRNA), and the result showed that hsa_circ_0068307 expression decreased significantly in both T24 and UMUC-3 cells (Fig. 1c). CCK8 detection (Fig. 1d, e) and colony formation assays (Fig. 1f, g) showed that hsa circ_0068307 knockdown suppressed T24 and UMUC-3 cell proliferation. Transwell assays showed that hsa circ_0068307 silencing decreased the migration ability of T24 and UMUC-3 cells (Fig. 1h, i). These data suggested that hsa_circ_0068307 is generally up-regulated in BCa clinical samples and cell lines, thus possesses a potential oncogenensis role in the progression of $\mathrm{BCa}$.

\section{Hsa_circ_0068307 functions as a miR-147 sponge, and c-Myc is a direct miR-147 target}

Next, we explored the hsa_circ_0068307 regulatory mechanism involved in $\mathrm{BCa}$ progression. Bioinformatics analysis was used to predict the hsa_circ_0068307 targets, which showed an interacting relationship between hsa_circ_0068307 and miR-147. WT or mutated sequences containing the miR-147 binding sequence were used to construct a luciferase reporter vector (Fig. 2a). The luciferase reporter vector was transfected into 293T cells, combined with or without the miR-147 mimic. Luciferase reporter analysis showed that miR147 inhibited the luciferase activity in WT cells, but not in mutated cell lines (Fig. 2b). This indicated that miR-147 was the target of hsa_circ_0068307. Rt-qPCR detection confirmed that hsa_circ_0068307 silencing suppressed hsa_circ_0068307 expression, and miR-147 inhibitor treatment failed to recover the expression of 

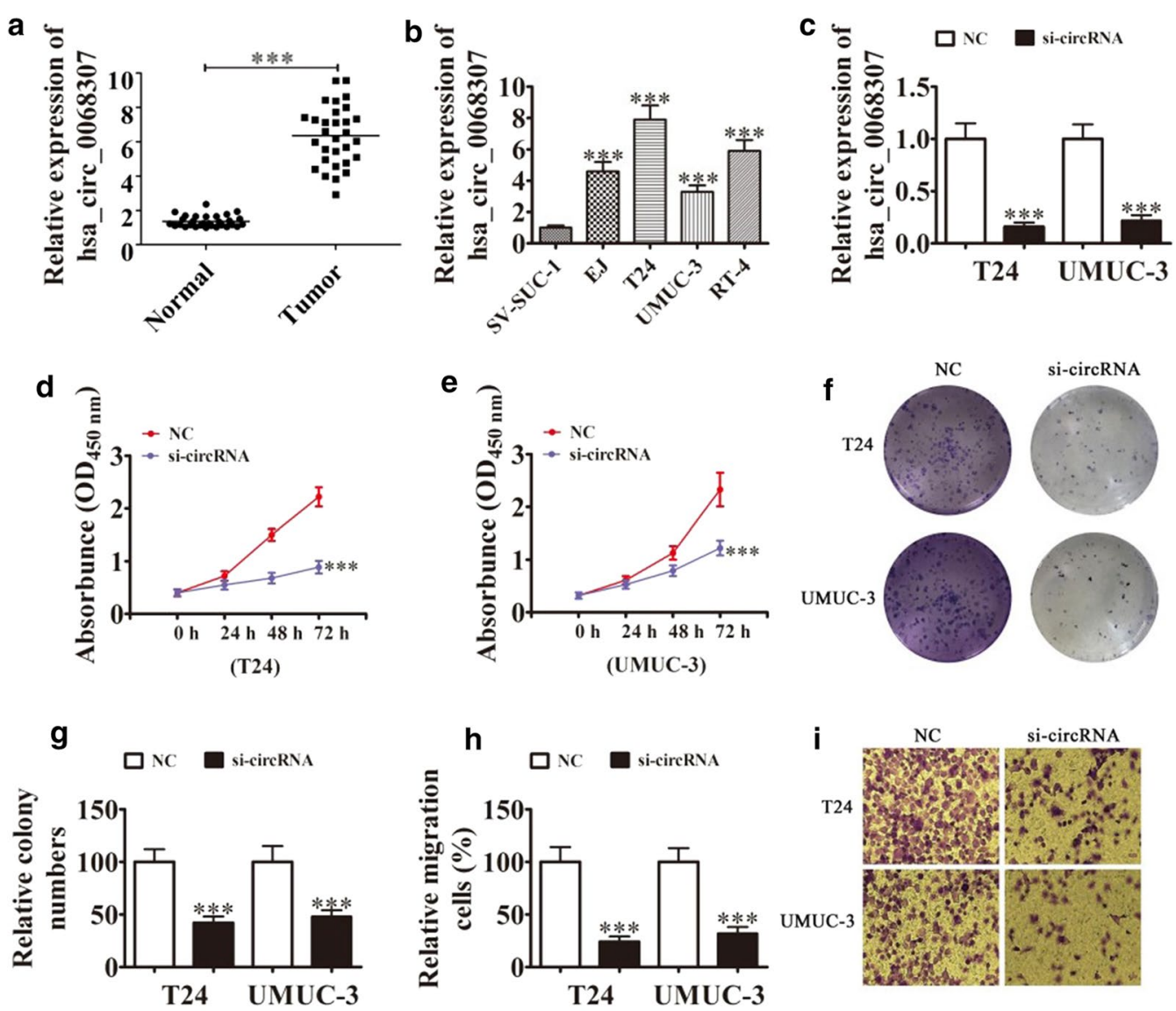

Fig. 1 Hsa_circ_0068307 is expressed at high levels in BCa and exerts oncogenic effects in the BCa cell lines T24 and UMUC3. a Rt-qPCR detection showing the expression of hsa_circ_0068307 in tumor tissues and adjacent normal tissues. Data are presented as the mean \pm SD. ${ }^{* * *} \mathrm{P}<0.001 . \mathbf{b}$ Rt-qPCR detection showing the expression of hsa_circ_0068307 in SV-HUC-1 and BCa cell lines. Data are presented as the mean $\pm S D$. ${ }^{* * * P}<0.001$ vs. SV-HUC-1. c The expression of hsa_circ_0068307 was detected in T24 and UMUC-3 cells transfected with siRNA hsa_circ_0068307 (si-circRNA) or negative control (NC). Data are presented as the mean \pm SD. ${ }^{* *} \mathrm{P}<0.001$ vs. NC. d, e CCK8 detection showing that hsa_circ_0068307 knockdown suppressed cell proliferation in T24 (d) and UMUC-3 (e) cells. Data are presented as the mean \pm SD. ${ }^{* * *}<0.001$ vs. NC. f, $\mathbf{g}$ Colony formation assays showing the proliferation of T24 and UMUC3 cells after knockdown of hsa_circ_0068307. Data are presented as the mean \pm SD. ${ }^{* * * P}<0.001$ vs. NC. $\mathbf{h}, \mathbf{i}$ Transwell assays showing the migration of BCa cells after knockdown of hsa_circ_0068307. Data are presented as the mean $\pm S D$. ${ }^{* * * P}<0.001$ vs. NC

hsa_circ_0068307 (Fig. 2c). However, hsa_circ_0068307 silencing upregulated miR-147 expression in UMUC-3 and T24 cells. miR-147 inhibitor treatment suppressed the promotion effect of hsa_circ_0068307 silencing (Fig. 2d). These results suggested that miR-147 was the hsa_circ_0068307 downstream target.

Bioinformatics analysis identified a relationship between miR-147 and the 3'-UTR of c-Myc. A mutated or WT sequence containing the miR-147 binding site was used to construct a luciferase reporter vector (Fig. 2e). The luciferase reporter vector was transfected into 293T cells, combined with or without the miR-147 mimic. The results of luciferase reporter assays showed that miR147 inhibited the luciferase activity in WT cells, but not in mutated cell lines (Fig. 2f), suggesting that miR-147 can interact with the $3^{\prime}$-UTR of c-Myc. Rt-qPCR detection confirmed that miR-147 overexpression (transfected with miR-147 mimics) upregulated miR-147 expression (Fig. 2g) and downregulated c-Myc (Fig. 2h). These results suggested that $\mathrm{c}-\mathrm{Myc}$ was the downstream target of miR-147.

\section{Knockdown of hsa_circ_0068307 inhibits cancer stem cell-mediated $\mathrm{BCa}$ cell proliferation and migration by regulating the $\mathrm{miR}-147 / \mathrm{c}-$ Myc axis}

Previous work focus on the regulative role of c-Myc in cell self-renewal process and stemness maintenance [13]. Si-hsa_circ_0068307 T24 cells show relatively poorer sphere formation ability compared to si-NC T24 cells (Additional file 1: Figure S1), which indicates 
a

hsa_circ_0068307-Wt $5^{\prime}$ cgucuGgUAUUUCCACACAc 3'
$:|:|||||||||||$ miR-147 3' cgucuUCGUAAAGGUGUGUg $5^{\prime}$ hsa_circ_0068307-Mut 5' cgucuGGUAAAAGGCACACc 3'

C

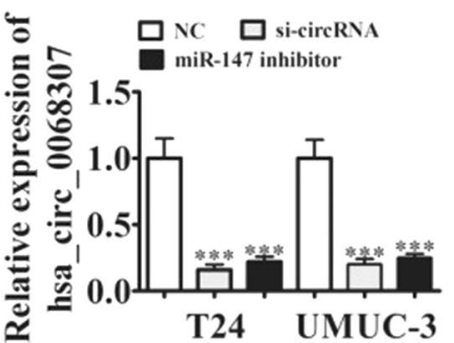

e

c-Myc-3'UTR-Wt $\quad 5$ ' ...AGAGGGAAGCUGAGGCACACAAA ... 3' || | | || || | miR-147 $33^{\prime} \quad$ CGUCUUCGUAAAGGUGUGUG

c-Myc-3'UTR-Mut $5^{\prime}$...AGAGgGaAgCUGAGgAgACACCA... 3'

\section{g}

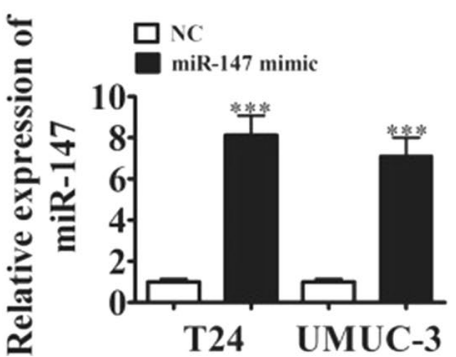

b

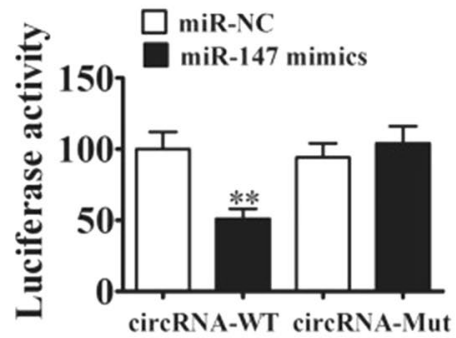

d

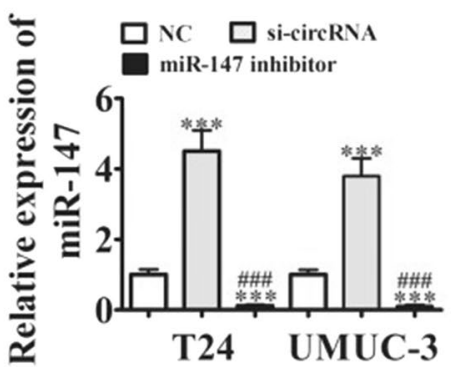

f

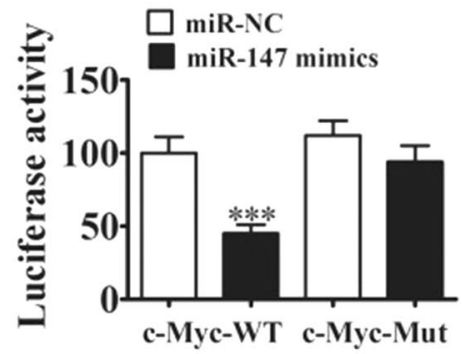

h

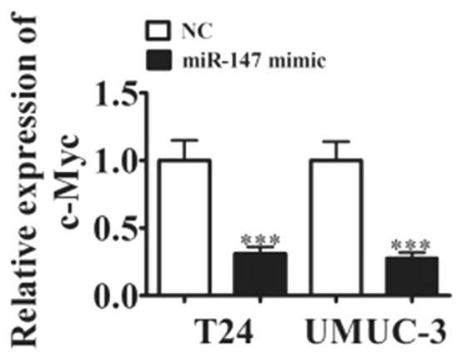

Fig. 2 Hsa_circ_0068307 acts as a sponge for miR-147, and c-Myc is a direct target of miR-147. a The complementary sites within hsa_circ_0068307 and miR-147 were predicted by bioinformatics analysis. The mutated (Mut) version of hsa_circ_0068307 is also shown. b Dual luciferase reporter assays demonstrated that miR-147 is a direct target of hsa_circ_0068307. Data are presented as the mean \pm SD. ${ }^{* * * P<0.001 ~ v s . ~ c o n t r o l . ~} \mathbf{c}$, d qRt-PCR detection showing the expression of hsa_circ_0068307 and miR-147 in T24 and UMUC3 cells transfected with si-circRNA or miR-147 inhibitor. Data are presented as the mean \pm SD. ${ }^{* *} \mathrm{P}<0.001 \mathrm{vs}$. NC. ${ }^{\# \#} \mathrm{P}<0.001 \mathrm{vs}$. si-circRNA. e The predicted binding sites of miR-147 with the $3^{\prime}-U T R$ of $c-M y c$. The mutated version of the $3^{\prime}$-UTR-c-Myc is also shown. $\mathbf{f}$ Relative luciferase activity was determined $48 \mathrm{~h}$ after transfection with miR-147 mimic/ normal control or with the $3^{\prime}$-UTR-c-Myc wild-type/Mut in HEK293T cells. Data are presented as the mean \pm SD. ${ }^{* * * P<0.001 ~ v s . ~ c o n t r o l . ~ g, ~} \mathbf{h}$ qRt-PCR detection showing the expression of miR-147 ( $\mathbf{g})$ and c-Myc (h) in T24 and UMUC3 cells transfected with miR-147 mimic. Data are presented as the mean $\pm S D$. ${ }^{* * *} \mathrm{P}<0.001$ vs. NC

hsa_circ_0068307 correlates with cell stemness ability. We further evaluated whether the proliferation suppression effect of silencing hsa_circ_0068307 was mediated via miR-147/c-Myc axis through several rescue experiments. RT-qPCR assays showed that hsa_circ_0068307 silencing suppressed hsa circ_0068307 and c-Myc expression and upregulated miR-147 expression. Combination treatment with the miR-147 inhibitor downregulated miR-147 expression and restored c-Myc expression. However, miR-147 
inhibitor treatment had no effect on hsa_circ_0068307 expression. Transfection with c-Myc overexpression vector significantly increased c-Myc in both T24 and UMUC-3 cells, whereas it had no effect on hsa circ_0068307 and miR-147 expression (Fig. 3a-f).
CCK8 detection (Fig. 3g, h) and colony formation assays (Fig. 3i, j) showed that miR-147 inhibitor treatment partially recovered hsa_circ_0068307 knockdown-induced decrease in proliferation ability. c-Myc overexpression increased the proliferation of both

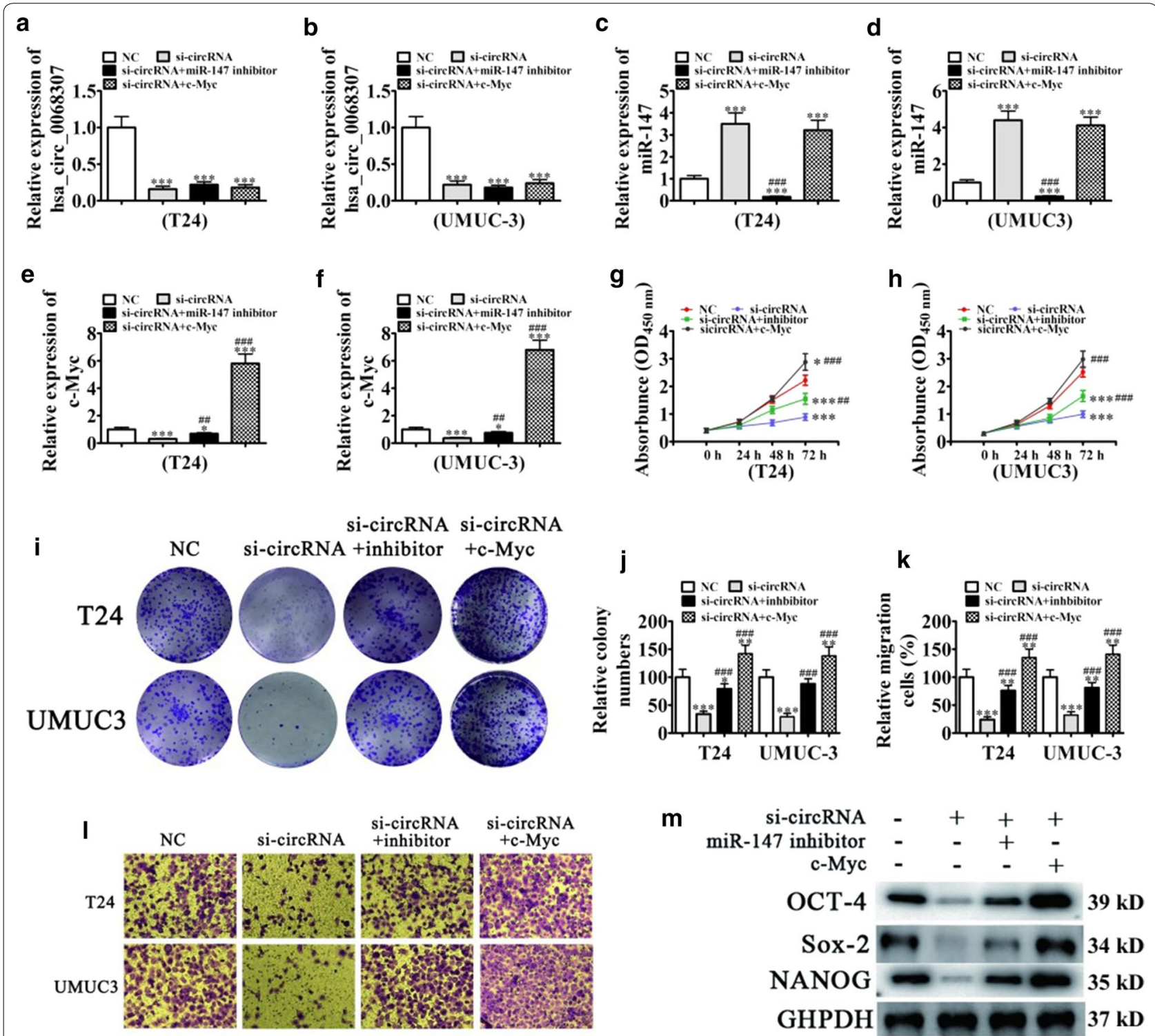

Fig. 3 Knockdown of hsa_circ_0068307 inhibits cancer stem cell-mediated BCa cell proliferation and migration by regulating the miR-147/c-Myc axis in in vitro. a, b RT-qPCR assay showing hsa_circ_0068307 expression in T24 (a) and UMUC3 (b) cells after transfection with siRNA against hsa_ circ_0000291, combined with or without miR-147 inhibitor or the c-Myc overexpression vector. Data are presented as the mean \pm SD. ${ }^{* * *}<0.001$ vs. NC. c, d RT-qPCR assay showing miR-147 expression in T24 (c) and UMUC3 (d) cells. Data are presented as the mean \pm SD. ${ }^{* * * P}<0.001$ vs. NC. $\# \#$ \# $<0.001$ vs. si-circRNA. e, $\mathbf{f}$ RT-qPCR detection showing c-Myc expression in T24 (e) and UMUC3 (f) cells. Relative protein levels were analyzed and data are presented as the mean \pm SD. ${ }^{* *} \mathrm{P}<0.001$ vs. NC. ${ }^{\# \#} \mathrm{P}<0.001$ vs. si-circRNA. $\mathbf{g}, \mathbf{h}$ CCK8 assays were performed to assess cell proliferation in $\mathrm{T} 24(\mathbf{g})$ and UMUC3 $(\mathbf{h})$ cells. Data are presented as the mean $\pm \mathrm{SD}$. ${ }^{*} \mathrm{P}<0.05$, ${ }^{* * *} \mathrm{P}<0.001 \mathrm{vs.} \mathrm{NC.}{ }^{\# \#} \mathrm{P}<0.001$ vs. si-circRNA. $\mathbf{i}$, j Clone formation assay showing the proliferation of T24 and UMUC3 cells. Data are presented as the mean \pm SD. ${ }^{* * P}<0.01$, ${ }^{* * *} \mathrm{P}<0.001$ vs. NC. $\# \# \#<0.001$ vs. Si-circRNA. $\mathbf{k}$, I Cell migration was determined in T24 and UMUC3 cells using Transwell ${ }^{\circledR}$ assays. Data are presented as the mean \pm SD. ${ }^{* *} P<0.01,{ }^{* * *}<<0.001$ vs. NC. $\# \#$ P $<0.001$ vs. si-circRNA. $\mathbf{m}$ Western blow analysis showing the expression of cancer stem cell-related proteins OCT-4, Sox-2, and NANOG in T24 and UMUC3 cells. Relative protein levels were analyzed and data are presented as the mean \pm SD. ${ }^{* * P}<0.001$ vs. NC. ${ }^{\# \#} P<0.001$ vs. si-circRNA 
UMUC-3 and T24 cells even after hsa_circ_0068307 silencing. Transwell assays also showed that miR147 inhibitor treatment partially recovered the hsa circ_0068307 knockdown-induced decrease in migration ability. c-Myc overexpression promoted migration in UMUC-3 and T24 cells even after hsa_circ_0068307 silencing (Fig. 3k, l). Western blot analysis showed that miR-147 inhibitor treatment partially recovered the expression of the CSC-related proteins, NANOG, OCT4, and Sox-2 after hsa_circ_0068307 silencing. c-Myc overexpression upregulated the expression of CSCrelated proteins in UMUC-3 and T24 cells even after hsa_circ_0068307 silencing (Fig. 3m).

\section{c-Myc overexpression reverses the miR-147 mediated} inhibition of cancer stem cell-mediated $\mathrm{BCa}$ cell migration and proliferation in vitro

We further validated the relationship between c-Myc and miR-147. We constructed miR-147 overexpressing cells with or without c-Myc overexpression. The results showed that miR-147 expression was increased in UMUC-3 and T24 cells transfected with the miR-147 mimic. However, c-Myc overexpression had no effect on miR-147 expression (Fig. 4a). miR-147 overexpression downregulated c-Myc in T24 and UMUC-3 cells transfected with miR-147 mimic. c-Myc overexpression significantly upregulated c-Myc because the heterogeneous transfection of c-My had no $3^{\prime}$-UTR and cannot interact with and be degraded by miR-147 (Fig. 4b).

CCK8 detection (Fig. 4c, d) and colony formation assays (Fig. 4e, f) showed that miR-147 overexpression decreased UMUC-3 and T24 cell proliferation, whereas c-Myc overexpression recovered and increased cell proliferation after miR-147 overexpression. Transwell assays showed that miR-147 overexpression decreased the migration ability of T24 and UMUC-3 cells. However, c-Myc overexpression promoted the migration of T24 and UMUC-3 cells, even after miR-147 overexpression
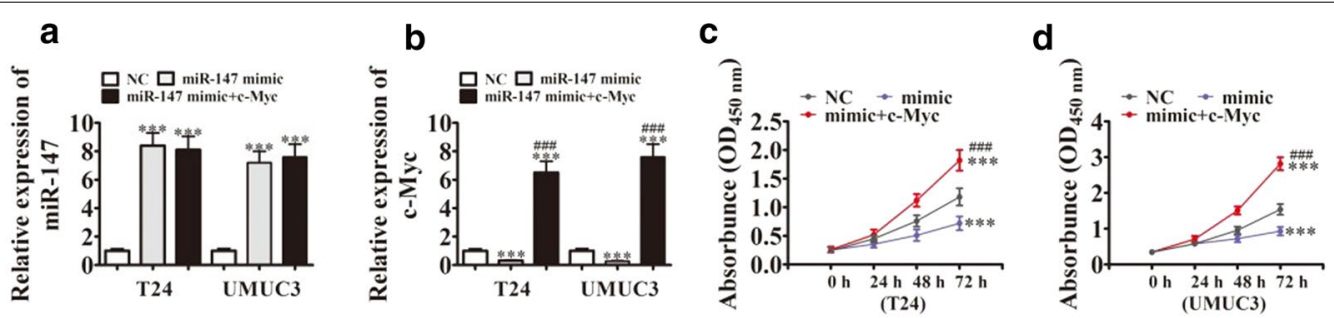

e

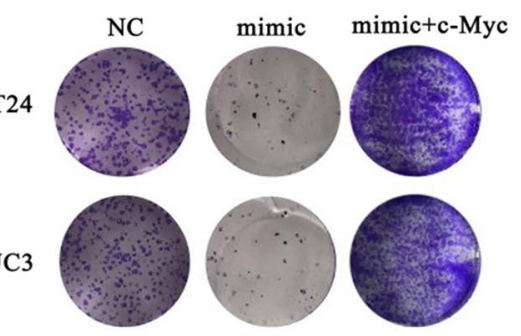

f

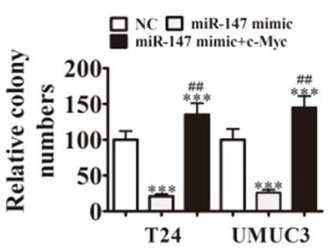

g
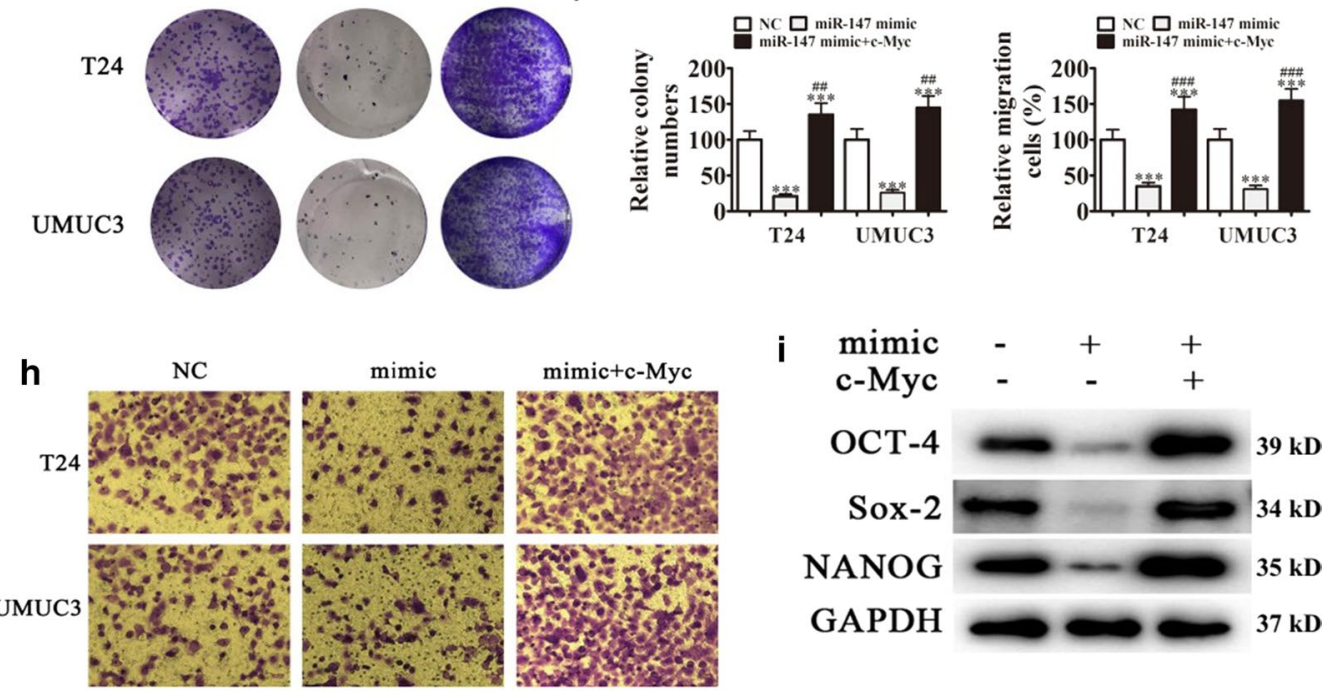

Fig. 4 C-Myc overexpression reverses the inhibitory effect of miR-147 on cancer stem cell-mediated BCa cell proliferation and migration in vitro. a, b qRT-PCR assay showing miR-147 (a) and c-Myc (b) expression in T24 and UMUC3 cells. Data are presented as the mean \pm SD. ${ }^{* * *}<0.001$ vs. NC. ${ }^{\# \# P}<0.001$ vs. si-circRNA. c, d CCK8 assays were performed to assess cell proliferation in T24 (c) and UMUC3 (d) cells. Data are presented as the mean $\pm S D$. ${ }^{*} P<0.05,{ }^{* *} P<0.001$ vs. NC. ${ }^{\# \#} \mathrm{P}<0.001$ vs. si-circRNA. e, fClone formation assay showing the cell proliferation of T24 and UMUC3 cells. Data are presented as the mean \pm SD. ${ }^{* *} \mathrm{P}<0.001$ vs. NC. ${ }^{\# \#} \mathrm{P}<0.01,{ }^{\# \#} \mathrm{P}<0.001$ vs. si-circRNA. g, $\mathbf{h}$ Cell migration was determined in T24 and UMUC3 cells using Transwell ${ }^{\circledR}$ assays. Data are presented as the mean \pm SD. ${ }^{* *} P<0.001$ vs. NC. ${ }^{\# \#} P<0.001$ vs. si-circRNA. i Western blot analysis showing the expression of cancer stem cell-related proteins OCT-4, Sox-2, and NANOG in T24 and UMUC3 cells 
(Fig. 4g, h). Western blot assays showed that miR-147 overexpression downregulated the CSC-related proteins, Sox-2, OCT-4, and NANOG, whereas c-Myc overexpression upregulated CSC-related proteins in T24 and UMUC-3 cells, even after miR-147 overexpression (Fig. 4i).

\section{Downregulation hsa_circ_0068307 suppresses tumor growth in nude mouse xenografts}

Finally, we validated the role of hsa_circ_0068307 involved in BCa in vivo. Hsa_circ_0068307 knockdown stable lentiviral strain (small hairpin RNA overexpression vector, sh-circRNA) or sh-NC T24 cells were used for tumor formation. The xenograft results confirmed that hsa_circ_0068307 knockdown suppressed tumor growth, as determined by tumor weight and volume, compared with the NC group (Fig. 5a-c). Rt-qPCR detection showed that hsa_circ_0068307 knockdown upregulated miR-147 expression in tumor tissues (Fig. 5d). Western blot detection showed that hsa_circ_0068307 knockdown downregulated c-Myc and the CSC-related proteins, NANOG, OCT-4, and Sox-2 in tumor tissues (Fig. 5e), which further validated the regulatory role of hsa_circ_0068307/miR-147/c-Myc in in vivo BCa xenograft model.

\section{Discussion}

Increasing evidence indicates that noncoding RNAs (such as circRNAs, miRNAs and lncRNAs) are aberrantly expressed in various cancers, and studies have focused on the role of epigenetic regulation in cancer development
$[19,20]$. The results of the present study suggested that hsa_circ_0068307 expression is increased in both Bca tissues and cell lines. Downregulation of hsa_circ_0068307 suppressed cell proliferation and migration in both T24 and UMUC-3 cells. These results indicate that hsa circ_0068307 is involved in BCa development and pathogenesis. To reveal the regulatory mechanism underlying the involvement of hsa_circ_0068307, we predicted that miR-147 and c-Myc were downstream targets of hsa circ_0068307 using bioinformatics analysis. Luciferase reporter assays confirmed that hsa_circ_0068307 regulated c-Myc expression by sponging miR-147.

miR-147 can suppresses human hepatocellular carcinoma migration, chemosensitivity, and proliferation by inhibiting HOXC6 [21]. miR-147 suppresses migration, proliferation, and invasion in breast cancer via the $\mathrm{AKT} / \mathrm{mTOR}$ signaling pathway [22]. In the present study, we showed that hsa_circ_0068307 silencing abolished the inhibitory effect of hsa_circ_0068307 on miR-147. Further study suggested that c-Myc was the target of miR-147.

Overexpression of c-Myc promotes the development of cancer [23, 24]. c-Myc contributes to maintain stemness, self-renewal and chemoresistant properties [25, 26]. The present results indicated that downregulation of c-Myc suppressed CSC differentiation in BCa. Moreover, overexpression $\mathrm{c}-\mathrm{Myc}$ increased the levels of stem cell markers, such as OCT-4, NANOG, and Sox-2. The positive regulation of self-renewal and the maintenance of stemness did not occur after silence hsa_circ_0068307, which decreased c-Myc level by promotion miR-147
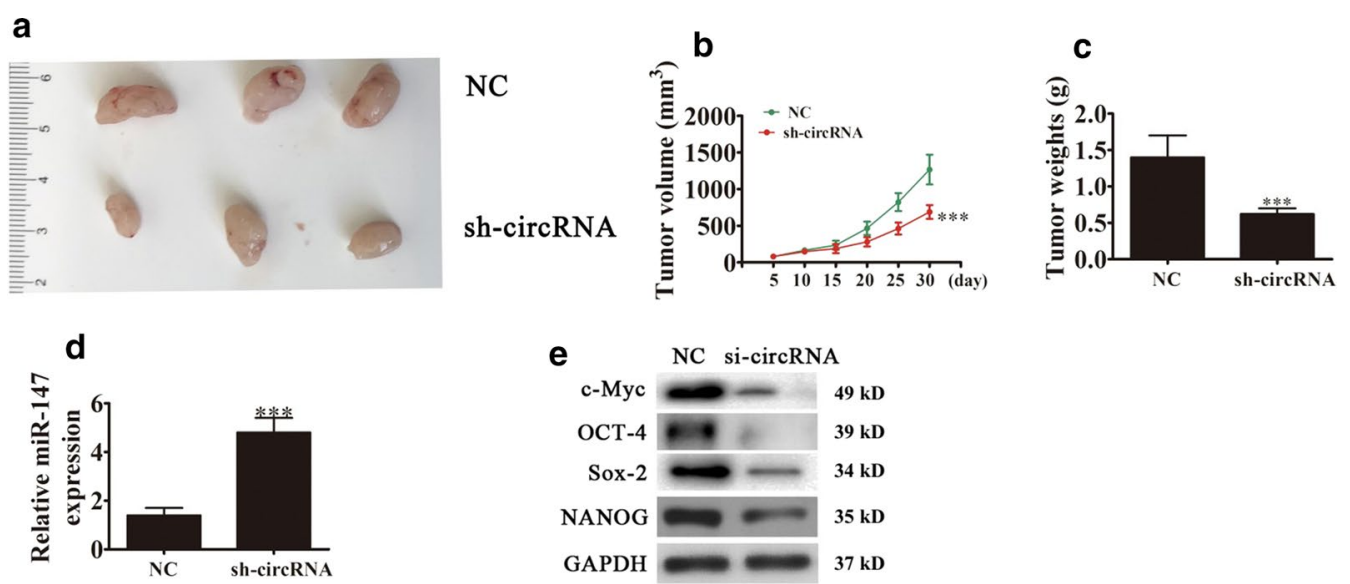

Fig. 5 Downregulation hsa_circ_0068307 suppresses tumor growth in nude mouse xenografts. a Representative photographs of T24 tumor formation in xenografts of nude mice. $n=6$ per group. $\mathbf{b}$ Summary of tumor volumes in mice measured every week. Data are presented as the mean $\pm S D .{ }^{* *} \mathrm{P}<0.01,{ }^{* *} \mathrm{P}<0.001$ vs. NC. c Tumor weight was measured after 30 days of injection. Data are presented as the mean $\pm \mathrm{SD}$. ${ }^{* * *} \mathrm{P}<0.001$ vs. NC. $\mathbf{d}$ qRT-PCR assay showing the expression of miR-147. Data are presented as the mean \pm SD. ${ }^{* *} \mathrm{P}<0.001$ vs. control. e Western blot analysis of the expression of c-Myc and cancer stem cell-related proteins OCT-4, Sox-2, and NANOG in tumor tissues. Data are presented as the mean $\pm S D$. ${ }^{* * *} \mathrm{P}<0.001 \mathrm{vs}$. NC 
expression. In conclusion, hsa_circ_0068307 promoted tumor growth in vitro and in vivo, primarily via sponging miR-147 and promotion c-Myc expression.

\section{Conclusions}

In conclusion, we demonstrated that hsa_circ_0068307 silencing suppressed the transcriptional activation of c-Myc by upregulating miR-147, which downregulated the CSC-related proteins, OCT-4, NANOG, and Sox-2 and led to decreased cell proliferation and migration. We provided several novel strategies for c-Myc inhibition. Additional studies would provide insight into the involvement of the hsa_circ_0068307/miR-147/c-Myc axis in tumorigenesis, which would provide novel therapeutic agents for the treatment of $\mathrm{BCa}$.

\section{Supplementary information}

Supplementary information accompanies this paper at https://doi. org/10.1186/s12935-020-01235-6.

Additional file 1. Images of tumor sphere formation assays in T24 cells (200 cells/well), scale bar, $100 \mu \mathrm{m}$.

\section{Abbreviations}

circRNAs: Circular RNAs; BCa: Bladder cancer; CSCs: Cancer stem cells; FBS: Fetal bovine serum; CCK-8: Cell Counting Kit-8; RT-qPCR: Quantitative reverse transcription-polymerase chain reaction.

\section{Acknowledgements}

We would like to thank the researchers and study participants for their contributions.

\section{Authors' contributions}

QC, QY and YM were performed all the experiments and analyzed the data; ZZ, SW, ZC, XC, HX and SJ carried out in all experiments and revised the manuscript; $\mathrm{HJ}$ and $\mathrm{CY}$ were designed the studies and prepared the manuscript with comments from all authors. All authors read and approved the final manuscript.

\section{Funding}

The study was supported by research grants from The Department level scientific research project of China (Grant No. SHDC12017X10).

\section{Availability of data and materials}

The datasets used and/or analyzed during the current study are available from the corresponding author on reasonable request.

\section{Ethics approval and consent to participate}

The study protocol was approved by the Ethics Committee of Huashan Hospital (Shanghai, China; approval no. KY2011-009) and conducted in accordance with the tenets of the Declaration of Helsinki. All patients consented to use of resected tissues for research purposes.

\section{Consent for publication}

Not applicable.

\section{Competing interests}

The authors declare that they have no competing interests.

\section{Author details}

${ }^{1}$ Department of Urology, Huashan Hospital, Fudan University, Shanghai, China. ${ }^{2}$ Fudan Institute of Urology, Huashan Hospital, Fudan University, Shanghai, China. ${ }^{3}$ Shanghai Mental Health Center, Shanghai Jiaotong
University School of Medicine, Shanghai, China. ${ }^{4}$ Department of Urology, Zhongshan Hospital, Fudan University, Shanghai, China. ${ }^{5}$ Shanghai Cancer Center, Fudan University, Shanghai, China. ${ }^{6}$ National Clinical Research Center for Aging and Medicine, Fudan University, Shanghai, China.

Received: 4 November 2019 Accepted: 28 April 2020

Published online: 06 May 2020

\section{References}

1. Antoni S, Ferlay J, Soerjomataram I, Znaor A, Jemal A, Bray F. Bladder cancer incidence and mortality: a global overview and recent trends. Eur Urol. 2017;71(1):96-108.

2. Mao W, Huang X, Wang L, Zhang Z, Liu M, Li Y, Luo M, Yao X, Fan J, Geng J. Circular RNA hsa_circ_0068871 regulates FGFR3 expression and activates STAT3 by targeting miR-181a-5p to promote bladder cancer progression. J Exp Clin Cancer Res. 2019;38(1):169.

3. Chen W, Zheng R, Zeng H, Zhang S, He J. Annual report on status of cancer in China, 2011. Chin J Cancer Res. 2015;27(1):2-12.

4. Chen LL, Yang L. Regulation of circRNA biogenesis. RNA Biol. 2015;12(4):381-8.

5. Greene J, Baird AM, Brady L, Lim M, Gray SG, McDermott R, Finn SP. Circular RNAs: biogenesis, function and role in human diseases. Front Mol Biosci. 2017:4:38.

6. Rybak-Wolf A, Stottmeister C, Glazar P, Jens M, Pino N, Giusti S, Hanan M, Behm M, Bartok O, Ashwal-Fluss R, et al. Circular RNAs in the mammalian brain are highly abundant, conserved, and dynamically expressed. Mol Cell. 2015;58(5):870-85.

7. Zheng Q, Bao C, Guo W, Li S, Chen J, Chen B, Luo Y, Lyu D, Li Y, Shi G, et al. Circular RNA profiling reveals an abundant circHIPK3 that regulates cell growth by sponging multiple miRNAs. Nat Commun. 2016;7:11215.

8. Zhao ZJ, Shen J. Circular RNA participates in the carcinogenesis and the malignant behavior of cancer. RNA Biol. 2017;14(5):514-21.

9. Yang P, Qiu Z, Jiang Y, Dong L, Yang W, Gu C, Li G, Zhu Y. Silencing of cZNF292 circular RNA suppresses human glioma tube formation via the Wnt/beta-catenin signaling pathway. Oncotarget. 2016;7(39):63449-55.

10. Xie H, Ren X, Xin S, Lan X, Lu G, Lin Y, Yang S, Zeng Z, Liao W, Ding YQ, et al Emerging roles of circRNA_001569 targeting miR-145 in the proliferation and invasion of colorectal cancer. Oncotarget. 2016;7(18):26680-91.

11. Zhuang C, Huang X, Yu J, Gui Y. Circular RNA hsa_circ_0075828 promotes bladder cancer cell proliferation through activation of CREB1. BMB Rep. 2020;53(2):82-7.

12. Li Y, Zheng F, Xiao X, Xie F, Tao D, Huang C, Liu D, Wang M, Wang L, Zeng $F$, et al. CircHIPK3 sponges miR-558 to suppress heparanase expression in bladder cancer cells. EMBO Rep. 2017;18(9):1646-59.

13. Wang T, Shigdar S, Gantier MP, Hou Y, Wang L, Li Y, Shamaileh HA, Yin W, Zhou SF, Zhao X, et al. Cancer stem cell targeted therapy: progress amid controversies. Oncotarget. 2015;6(42):44191-206.

14. Chen B, Zhu Z, Li L, Ye W, Zeng J, Gao J, Wang S, Zhang L, Huang Z. Effect of overexpression of Oct4 and Sox2 genes on the biological and oncological characteristics of gastric cancer cells. Onco Targets Ther. 2019;12:4667-82

15. Zhu YJ, Zheng B, Luo GJ, Ma XK, Lu XY, Lin XM, Yang S, Zhao Q, Wu T, Li ZX, et al. Circular RNAs negatively regulate cancer stem cells by physically binding FMRP against CCAR1 complex in hepatocellular carcinoma. Theranostics. 2019;9(12):3526-40

16. Xiao T, Xue J, Shi M, Chen C, Luo F, Xu H, Chen X, Sun B, Sun Q, Yang Q, et al. Circ008913, via miR-889 regulation of DAB2IP/ZEB1, is involved in the arsenite-induced acquisition of CSC-like properties by human keratinocytes in carcinogenesis. Metallomics. 2018;10(9):1328-38.

17. Li R, Wu B, Xia J, Ye L, Yang X. Circular RNA hsa_circRNA_102958 promotes tumorigenesis of colorectal cancer via miR-585/CDC25B axis. Cancer Manag Res. 2019;11:6887-93.

18. Yang D, Du G, Xu A, Xi X, Li D. Expression of miR-149-3p inhibits proliferation, migration, and invasion of bladder cancer by targeting S100A4. Am J Cancer Res. 2017;7(11):2209-19.

19. Hosono Y, Niknafs YS, Prensner JR, lyer MK, Dhanasekaran SM, Mehra R, Pitchiaya S, Tien J, Escara-Wilke J, Poliakov A, et al. Oncogenic role of THOR, a Conserved cancer/testis long non-coding RNA. Cell. 2017;171(7):1559-1572.e1520. 
20. Su H, Tao T, Yang Z, Kang X, Zhang X, Kang D, Wu S, Li C. Circular RNA CTFRC acts as the sponge of MicroRNA-107 to promote bladder carcinoma progression. Mol Cancer. 2019;18(1):27.

21. Sui CJ, Xu F, Shen WF, Dai BH, Lu JJ, Zhang MF, Yang JM. MicroRNA-147 suppresses human hepatocellular carcinoma proliferation migration and chemosensitivity by inhibiting HOXC6. Am J Cancer Res. 2016;6(12):2787-98

22. Zhang Y, Zhang HE, Liu Z. MicroRNA-147 suppresses proliferation, invasion and migration through the AKT/mTOR signaling pathway in breast cancer. Oncol Lett. 2016;11(1):405-10.

23. Li H, Liu J, Cao W, Xiao X, Liang L, Liu-Smith F, Wang W, Liu H, Zhou P, Ouyang R, et al. C-myc/miR-150/EPG5 axis mediated dysfunction of autophagy promotes development of non-small cell lung cancer. Theranostics. 2019;9(18):5134-48.

24. Chang YT, Teng YN, Lin KI, Wang CCN, Morris-Natschke SL, Lee KH, Hung CC. Danazol mediates collateral sensitivity via STAT3/Myc related pathway in multidrug-resistant cancer cells. Sci Rep. 2019;9(1):11628.
25. Zhang HL, Wang P, Lu MZ, Zhang SD, Zheng L. c-Myc maintains the self-renewal and chemoresistance properties of colon cancer stem cells. Oncol Lett. 2019;17(5):4487-93.

26. Kubota S, Tokunaga K, Umezu T, Yokomizo-Nakano T, Sun Y, Oshima $\mathrm{M}$, Tan KT, Yang H, Kanai A, Iwanaga E, et al. Lineage-specific RUNX2 super-enhancer activates MYC and promotes the development of blastic plasmacytoid dendritic cell neoplasm. Nat Commun. 2019;10(1):1653.

\section{Publisher's Note}

Springer Nature remains neutral with regard to jurisdictional claims in published maps and institutional affiliations.
Ready to submit your research? Choose BMC and benefit from:

- fast, convenient online submission

- thorough peer review by experienced researchers in your field

- rapid publication on acceptance

- support for research data, including large and complex data types

- gold Open Access which fosters wider collaboration and increased citations

- maximum visibility for your research: over 100M website views per year

At BMC, research is always in progress.

Learn more biomedcentral.com/submissions 